are transferred to the patient's eye. A much more simple method is that of Klein, who uses as his fixation light on the perimeter a filament lamp which rotates with the arc, the image of which is then projected upon the eye, see Duke-Elder's Text-Book of Ophthalmology, Vol. II.

I take the liberty to offer a new simple method, founded on keratoscopic examination, Fig. 1. Instead of concentric rings across the centre of the disc is drawn a black line, which passes through the zero, marked on the back of the disc. Here is placed, similar to the keratoscope of Weve, a metallic rod with weighta plummet-fastened to the movable frame of the magnifyingglass, $6.0 \mathrm{D}$., placed in the hole of the disc. The rod determines a perpendicular, $0^{\circ}$, or a declination.

The proceeding is simple: we set the metallic rod suitable to the site of the arc of the perimeter, in the Fig. $52^{\circ}$ from the horizontal, and holding the disc parallel to the iris or suspending it on the free end of the rod at a distance of about $12 \mathrm{~cm}$., the diameter of the disc is $33 \mathrm{~cm}$., we look at the corneal image. The image of the centre of the disc shall be brought to the centre of the cornea. The image of the black line indicates the meridian of longitude, determined by perimetric measurement, in which the retinal landmark is situated. The two points on opposite sides of the limbus are marked with some indicator.

\title{
ANNOTATION
}

\section{Recent changes in some Continental Ophthalmological Journals}

The Zeitschrift für Augenheilkunde now appears as "Ophthalmologica." It is published as an international journal of ophthalmology at Basel and contains articles in English, French and German. The list of editors is world-wide and we note that for Britain these are A. J. Ballantyne, Sir S. Duke-Elder, Mr. C. B. Goulden and Miss Ida Mann. We understand that the Archiv. für Ophthalmologie and the Archiv. für Augenheilkunde have been recently amalgamated. We do not know what facts underlie these changes. The causes of amalgamation of the two Archiv. may possibly be financial. Like Mr. Squeers, when Bolder's father was two pound ten short, they may have had disappointments to contend against. The financing of an ophthalmological journal is never an easy matter in any country. Union usually means strength and under conditions as they are to-day in Central Europe there may not be sufficient material to enable two large journals such as the 
two Archiv. to keep going. If so an amalgamation is the obvious way in which to meet the difficulty. But for the Zeitschrift to change its name and country of publication appears to us to suggest that the editorial department feel that they need a freer hand in matters for publication relating to their own subject. It will be a poor day for science of every kind if methods of control are allowed to interfere with the free interchange of knowledge; for science is truly international and independent of race and creed.

\section{ABSTRACTS}

\section{I.-MEDICAL OPHTHALMOLOGY}

(1) Naffziger, Howard C. (San Francisco).-Progressive exophthalmos associated with disorders of the thyroid gland. Annals of Surgery, October, 1938.

(1) Naffziger states that before 1931 a review of the literature showed no concurrence of opinion as to the reasons for the progressive exophthalmos occasionally seen following thyroidectomy. He then had a case in which operative treatment was successful and he was able at the same-time to investigate the pathological changes involved. Since then a number of other cases have been investigated and the pathological changes have been verified by numerous observers.

"Granting that our use of the term exophthalmos is loose and inaccurate, one cannot say much more than that, after an operation for goitre, the apparent exophthalmos disappears in about one-half of the cases and lessens in an additional 15 or 20 per cent. In others it may remain unchanged, but in a few there will be progression of varying grades. This progression may continue slowly, causing no more difficulty than that involved in an unsightly appearance of the patient ; progression may halt at any time and it is, fortunately, in only a very small number that it will progress to a danger point."

The cases in which operation has been performed by other surgeons together with eight of the author's make a total of 31 . In this series two deaths occurred and four results were considered poor. In the author's series of eight cases the age varied from 28 to 53 years. Four were females, four males. All had marked thyrotoxicosis at some time.

Examination of pieces of tissue taken from the orbits at the time of operation proves that the ocular muscles show varying degrees of degeneration, fibrosis and cellular infiltration. 